\title{
Lack of value of routine analysis of cerebrospinal fluid for prediction and diagnosis of external drainage-related bacterial meningitis
}

\author{
Rogier P. Schade, M.D., Ph.D., Janke Schinkel, M.D., Ph.D., Freek W. C. Roelandse, \\ Ronald B. Geskus, Ph.D., Leo G. Visser, M.D., Ph.D., Marc C. van DiJK, M.D., Ph.D., \\ Joan H. C. Voormolen, M.D., Ph.D., Hans van Pelt, and Ed J. KuiJPer, M.D., Ph.D.
}

Departments of Medical Microbiology, Clinical Chemistry, Medical Statistics, Infectious Diseases, and Neurosurgery, Leiden University Medical Center, Leiden, The Netherlands

\begin{abstract}
Object. Routine microbiological and chemical analysis of cerebrospinal fluid (CSF) is often performed to diagnose external drainage-related bacterial meningitis (ED-BM) at an early stage. A cohort study was performed to investigate the value of several commonly used CSF parameters for the prediction and diagnosis of ED-BM.

Methods. In a cohort of 230 consecutive patients in whom external drains had been placed, CSF samples were collected daily, prospectively evaluated for the presence of bacteria using Gram stain and microbiological culture, and analyzed for leukocyte count, protein concentration, glucose concentration, and ratio of CSF glucose to blood glucose. In addition, the CSF concentration of interleukin-6 (IL-6) was determined. The definition of ED-BM was based on positive culture results in combination with clinical symptoms. A matched case-control study was performed to evaluate the cohort longitudinally and to control for biasing factors such as duration of external drainage.

External drainage-related bacterial meningitis developed in 22 patients (9.6\%). Results from analyses of $1516 \mathrm{CSF}$ samples showed no significant differences between the patients in whom ED-BM developed and a control group without ED-BM during the first 3 days of infection or during the 3 days preceding the infection with regard to leukocyte count, protein concentration, glucose concentration, and CSF/blood glucose ratio. No significant difference between groups was found for the CSF IL- 6 concentration during the 3 days preceding the infection. In the matched case-control study, none of the parameters had significant predictive or diagnostic value for ED-BM in analyses using absolute values, ratios, and differences between the current and previous day's values. A comparison of the results from Gram stains and CSF cultures showed that the Gram staining had a very high specificity (99.9\%) but a low sensitivity (18\% [four of 22 patients] on the 1 st day of infection and $60 \%$ [nine of 15 patients] on the 2 nd day).

Conclusions. Severe disturbances in the CSF of patients with external drains limit the value of routine CSF analysis for prediction or diagnosis of ED-BM. Routine Gram stain of CSF has also limited predictive or diagnostic value due to its low sensitivity in screening for ED-BM.
\end{abstract}

\section{KEY WORDS • bacterial meningitis • cerebrospinal fluid drainage • central nervous system infection}

$\mathrm{E}$ XTERNAL drainage of CSF is a frequently performed procedure in the neurosurgical department. Common indications are transient hydrocephalus in relation to intracranial hemorrhage or tumors, the prevention of CSF fistulas after neurosurgical procedures, and the monitoring of intracranial pressure. One of the major complications is ED-BM; reported infection rates vary between 3 and $20 \%$. 1,4,5, ,14,15,21-23 Risk factors for the development of ED-BM are duration of drainage and drain-related factors such as manipulation and site leakage. ${ }^{13,20}$ To facilitate the diagnosis of ED-BM in patients with CSF drainage, routine analysis of CSF is often performed. In patients with bacterial meningitis that is not related to external drainage, an increased leukocyte count and protein concentration and a decreased glucose concentration are highly predictive of the presence of

Abbreviations used in this paper: $\mathrm{CSF}=$ cerebrospinal fluid; $\mathrm{ED}-$ $\mathrm{BM}=$ external drainage-related bacterial meningitis; $\mathrm{IL}=$ interleukin; $\mathrm{ROC}=$ receiver operating characteristic. this infection. In patients in whom an external drain has been placed, however, the composition of CSF is not comparable to that in individuals not undergoing external drainage, due to underlying disease and/or the effects of neurosurgical procedures in patients with external drains. ${ }^{3,6,19}$ Although CSF analysis is often used to screen for ED-BM, it is currently unknown whether CSF analysis can be used to predict bacterial meningitis in patients undergoing external drainage. Microbiological tests remain the gold standard for the diagnosis of ED-BM, but they are usually more timeconsuming than chemical analysis and leukocyte counts. It would therefore be useful to establish cutoff values for CSF parameters that predict the development or existence of bacterial meningitis in patients with external drains.

We evaluated a cohort of 230 patients who had external drains in whom daily CSF samples were obtained. These samples were evaluated using Gram stain and microbiological culture and analyzed for protein and glucose concentrations and leukocyte count. The ED-BM was defined 
based on positive culture results in combination with clinical symptoms. External drainage-related bacterial meningitis developed in 22 patients $(9.6 \%)$ in this cohort. We examined the predictive and diagnostic value of the CSF analysis and Gram stain for ED-BM. In a transversal analysis, the results of the Gram stain and CSF analysis for the 22 patients on the days before and during the infection were compared with those for the patients with external drains in whom ED-BM did not develop. Subsequently, we performed a longitudinal analysis using a matched case-control study in which the case group was matched for biasing factors such as duration of external drainage and the presence of blood in the CSF.

\section{Clinical Material and Methods}

\section{Patient Population}

A consecutive single-center cohort of 280 patients who had been undergoing external drainage of CSF for more than 24 hours between July 1999 and January 2003 was considered for inclusion in this study. During the period of treatment, CSF samples were obtained daily in each patient. Culture and Gram stain of the CSF samples were performed daily, and general CSF analysis (determination of leukocyte count and protein and glucose concentrations) was done every weekday. Clinical records were examined for patient characteristics, clinical signs (fever, signs of meningitis, headache, neurological status), drain characteristics (blockage, site leakage, involuntary disconnection), diagnostic test results, and treatment information. All clinical data were recorded on standard data entry forms and subsequently entered into a database. The study was approved by the Ethics Review Committee of the Leiden University Medical Center.

\section{Definition of Infection and Exclusion Criteria}

Infections related to external drainage were defined using microbiological data acquired from daily CSF monitoring in combination with clinical data. ${ }^{13}$ We defined ED-BM as a positive CSF culture on 1 or more consecutive days in combination with one or more clinical signs of bacterial meningitis (fever, headache, nuchal rigidity, and/or altered mental status). ${ }^{20}$ The 1 st day on which a positive culture was obtained was considered the 1st day of the diagnosed infection. If a patient had two or more consecutive positive CSF cultures with the same pathogen but no clinical symptoms, the result was defined as bacterial colonization of the drainage catheter. If a patient had only one positive CSF culture with a common skin pathogen, the results of subsequent consecutive samples were negative, and no treatment had been started, then the positive CSF result was considered a contamination. Contamination was identified in 20 patients. Patients with negative cultures or contaminated cultures were defined as not having ED-BM.

Excluded from the study were 30 patients with temporarily externalized internal CSF drains, such as malfunctioning ventriculoperitoneal or ventriculoatrial drains. Twenty patients with a central nervous system infection, such as cerebral abscess or tuberculosis, that was present before placement of the drain were also excluded. Therefore, 230 patients were included in the final analysis.

In 12 patients, external drainage was interrupted for more than 24 hours before a new drain was inserted, and the period after the interruption was considered a new external drainage period. We analyzed data for only the first external drainage period of each patient included in the study.

\section{Insertion and Maintenance of Drainage Catheters}

Placement of the drainage catheters occurred in the operating room or under sterile conditions at the patient's bedside. For placement of ventricular catheters, the patient's skull was shaved and prepared with standard sterile techniques. A small incision was made over the coronal suture in line with the pupil of the ipsilateral eye. A 6-mm bur hole was made through the skull, and the dura was incised with a small knife. The catheter was inserted in the ipsilateral ventricle. The wound was closed with sutures, and the catheter was sewn to the scalp to prevent dislodgment. For placement of lumbar catheters, a spinal catheter was inserted in the lower lumbar subarachnoid space using a Tuohy needle. The remaining part of the catheter was positioned transversely across the back and slightly up alongside of the abdomen.

For drainage of CSF using both ventricular and lumbar catheters, a closed external drainage and monitoring system (Exacta; Medtronic, Inc., Minneapolis, MN) was connected to the catheter. The CSF samples were obtained from this closed system through a valve that is part of the drainage system.

All patients who had undergone craniotomy before placement of the drainage catheter or during external drainage received $1 \mathrm{~g}$ of flucloxacillin intravenously before surgery. In cases of transsphenoidal or transsinusoidal surgery, $1 \mathrm{~g}$ of cefazolin was given intravenously. No special antibiotic agents were given prophylactically to patients whose drainage catheters were inserted at bedside. The patients did not receive prophylactic antibiotics during the period of external drainage.

\section{Microbiological Testing}

The CSF samples were sent daily to the laboratory for direct examination, Gram staining, and microbiological culture. The CSF was centrifuged, and the sediment was Gram stained and cultured on 5\% sheep blood agar; chocolate agar; and cysteine-, lactose-, and electrolyte-deficient agar for 5 days at $35^{\circ} \mathrm{C}$ in a $5 \% \mathrm{CO}_{2}$-enriched environment. Sediments were also cultured in fluid enrichment media. Bacteria were identified using standard biochemical tests. ${ }^{16}$ Antibiotic susceptibility testing was performed using an automated system (VITEK 2; bioMérieux, Marcy l'Etoile, France). The results of the susceptibility tests were used for comparison of the isolates.

\section{Leukocyte Count and Routine Chemical Analysis}

Cell counts of leukocytes and erythrocytes were determined in native samples using the Fuchs-Rosenthal counting chamber and light microscopy. The samples were centrifuged at $1900 \mathrm{G}$ for 10 minutes at room temperature, and the total protein and glucose concentrations of the supernatants were measured using an automated chemistry analyzer (Hitachi 911; Roche Diagnostics GmbH, Mannheim, Germany). For this study, leukocyte counts were presumed to be representative for the leukocyte count after cor- 
rection for red blood cell count by subtracting one point for each 1000 red blood cells.

\section{Concentrations of IL-6}

After determination of the total protein and glucose concentrations, the supernatants were frozen at $-20^{\circ} \mathrm{C}$ until further analysis. The levels of IL- 6 were determined by an enzyme-linked immunosorbent assay (PeliKine compact human IL-6 kit; Central Laboratory of the Netherlands Red Cross, Amsterdam, The Netherlands). Absorbencies were measured using a microplate spectrophotometer (Spectramax 250; Molecular Devices, Inc., Sunnyvale, CA). For values higher than the IL-6 assay cutoff of $3600 \mathrm{pg} / \mathrm{ml}$, we used the value of $3600 \mathrm{pg} / \mathrm{ml}$ in the analyses.

\section{Statistical Analysis}

Clinical and laboratory data were analyzed using statistical software (SPSS, version 11.0; SPSS, Inc., Chicago, IL). To determine the predictive and/or diagnostic value of the CSF leukocyte count, protein and glucose concentrations, and the CSF/blood glucose ratio for ED-BM, two analyses were performed. First, results obtained in all 22 patients with ED-BM were tested against those in 200 patients without ED-BM by using the Student t-test. Subsequently, a matched case-control study was performed to control the CSF results for biasing factors. The 22 patients with ED$\mathrm{BM}$ were matched for indication for external drainage, presence of blood in the CSF, and duration of external drainage with 22 control patients without bacterial meningitis. To determine the predictive value of each CSF parameter on the 3 days before the infection and the diagnostic value on the first 3 days of infection, univariate conditional logistic regression analysis was performed. In addition, the CSF parameter results were evaluated using ROC curves. To analyze the predictive and diagnostic value of a combination of CSF parameters, a descriptive analysis was performed, and the results were subsequently tested using the McNemar exact test. For all statistical analyses, a probability value less than 0.05 was considered significant.

\section{Results}

\section{Description of the Study Group}

Two hundred thirty patients were included in the study. The median patient age was 52.5 years (range $0.1-87.6$ years). The male/female ratio was 1:1.3. Lumbar catheters were placed in 125 patients $(54.3 \%)$, ventricular catheters in $97(42.2 \%)$, and more than one type of catheter in eight $(3.5 \%)$. The most frequent indications for external drainage were prevention and/or treatment of CSF leakage in 124 patients $(53.9 \%)$ and intracranial hemorrhage in 83 patients $(36.1 \%)$. The median duration of external drainage was 6 days (range $2-47$ days).

Twenty-two patients (9.6\%) had ED-BM. Gram-positive bacteria were isolated in $18(81.8 \%)$ of the 22 patients with ED-BM. Coagulase-negative staphylococci were found in eight, Staphylococcus aureus in six, Enterococcus faecalis in two, coagulase-negative staphylococci in combination with $S$. aureus in one, and Bacillus cereus in one patient. Gram-negative pathogens were encountered in four patients (18.2\%) with ED-BM. Proteus mirabilis, Pseudomonas aeruginosa, Klebsiella pneumoniae, and Escherichia coli were found in one patient each.

Bacterial colonization of the drainage catheter was found in eight patients $(3.5 \%)$ with external drainage. Gram-positive bacteria were found in six $(75.5 \%)$ of these eight patients. Coagulase-negative staphylococci were found in three patients, Corynebacterium spp. in one patient, and coagulase-negative staphylococci in combination with $S$. aureus in one patient and in combination with Corynebacterium spp. in one patient. The drainage catheter was colonized with Enterobacter cloacae in two patients.

The median time from the start of external drainage until the occurrence of infection was 6 days (range 2-17 days). All patients with ED-BM were febrile during the period of positive CSF cultures. In seven patients (31.8\%), accompanying signs of meningitis, including headache, nuchal rigidity, and/or altered mental status, were present. In one patient $(4.5 \%)$, signs of infection at the drainage site were also present.

Fifteen patients $(68.2 \%)$ with ED-BM had two or more consecutive positive CSF cultures. In seven patients $(31.8 \%)$, only one culture was positive, but by the time of subsequent cultures, treatment had already been initiated with antibiotic agents to which the cultured pathogen was susceptible.

All patients with ED-BM received antibiotic treatment. Of 22 patients, one $(4.5 \%)$ died due to the consequences of the cerebral infection with $K$. pneumoniae. Treatment had been started in the patient after clinical symptoms had developed and a positive Gram stain of the patient's CSF was obtained. The patient died the following day of complications of raised intracranial pressure. Postmortem examination showed signs of acute encephalitis secondary to bacterial meningitis.

\section{Predictive and Diagnostic Value of CSF Parameters}

A total of $1516 \mathrm{CSF}$ samples were analyzed for leukocyte count, protein and glucose concentrations, and CSF/blood glucose ratio. Figure 1 shows results for these parameters in 10 representative patients with ED-BM and 10 representative control patients during the period of external drainage. Figure 2 shows median results for all parameters in the 22 patients with ED-BM on the 3 days before infection and the first 3 days of infection, compared with the median results for the 200 patients without ED-BM. Statistical analysis showed no significant differences between the patients with ED-BM and the control patients on each of these days for all parameters $(\mathrm{p}>0.05$, Student t-test).

In the matched case-control study, we first performed a univariate conditional logistic regression analysis to determine the predictive value of each CSF parameter during the 3 days preceding the infection and the diagnostic value during the first 3 days of infection. We evaluated the value of the absolute level of the parameter; the difference in absolute level, compared with the absolute level on the day before (or 2 days before); and the ratio of the absolute level and the level on the day before (or 2 days before). No statistically significant differences between groups were found in any of these analyses (data not shown).

Second, we evaluated the predictive and diagnostic value of the CSF parameters using ROC curves. For none of the CSF parameters was it possible to establish a cutoff val- 

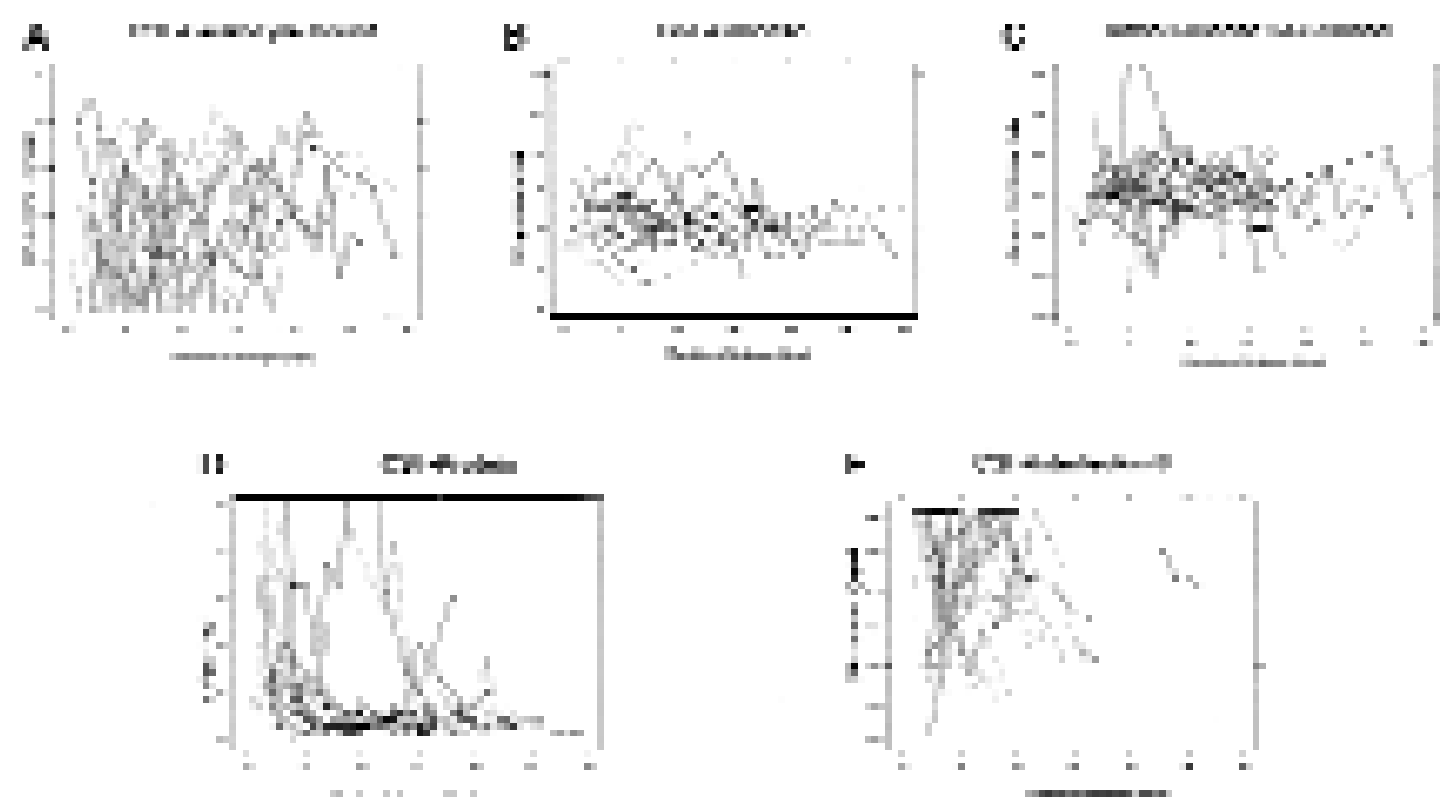

FIG. 1. Graphs showing representative measurements of CSF leukocyte count (A), CSF glucose concentration (B), CSF/blood glucose ratio (C), CSF protein concentration (D), and CSF IL-6 concentration (E) in 10 patients with external drains and ED-BM (heavy lines) and 10 patients with external drains but no ED-BM (thin lines). Representative results were selected based on the quantiles of the mean value; in each quantile the patient with the most measurements is depicted.

ue with a sensitivity and specificity of at least $60 \%$. Figure 3 shows examples of the ROC curves for the 1st day of infection.

Finally, a descriptive analysis was performed to evaluate the predictive and diagnostic value of combinations of CSF parameters. For each parameter, we established abnormal levels based on the 5th and 95th percentiles of the CSF results of 994 samples from the 200 patients without ED-BM. The abnormal levels were greater than 2705 for leukocyte count (95th percentile), less than $2.7 \mathrm{mmol} / \mathrm{L}$ for glucose concentration (5th percentile), greater than $2.34 \mathrm{~g} / \mathrm{L}$ for protein concentration (95th percentile), and less than 0.38 for the CSF/blood glucose ratio (5th percentile). Table 1 shows the number of patients with ED-BM and matched control patients with an abnormal result for one or more of the parameters. Few patients in the case group had abnormal values for two or more parameters both during the period before infection and during ED-BM. No statistically significant differences were found between the case group and the control group.

To evaluate the predictive and diagnostic value of the CSF parameters in patients with ED-BM caused by Gramnegative bacteria, all previously described analyses were also performed for a subgroup of four patients with Gramnegative ED-BM and matched control patients. No statistically significant differences between the two groups were found.

All previously described analyses were also performed for the group of 150 patients who did not have blood in the CSF. No statistically significant differences were found between patients with and without ED-BM.

\section{Predictive and Diagnostic Value of IL-6 Concentration}

One hundred sixty-six CSF samples from 21 patients with external drains could be evaluated for IL- 6 concentration. There was a significant correlation between IL- 6 concentrations and CSF leukocyte counts $(r=0.28, p<0.001)$. Figure 2 shows median IL-6 levels in 10 patients with ED$\mathrm{BM}$ on the 3 days before infection and the first 3 days of infection, compared with the median of all results obtained in 11 patients without ED-BM. There were no statistically significant differences between the patients with ED$\mathrm{BM}$ and the control patients on the 3 days before infection and on the 1st day of infection. Significant differences were found for the 2nd and 3rd days of infection. In a conditional logistic regression analysis of data for 10 case-control pairs, no significant differences between groups were found for IL-6 concentration.

\section{Diagnostic Value of the Gram Stain}

The Gram stain was used to evaluate 1735 CSF samples. Thirty-nine samples (2.2\%) showed a positive Gram stain. Compared with a positive culture, the overall sensitivity of the Gram stain was 39.8\% (38 of 98) and the specificity was $99.9 \%$ (1636 of 1637). The positive predictive value of the Gram stain was $97.4 \%$, and the negative predictive value was $96.5 \%$.

The Gram stain findings for Gram-positive and Gramnegative bacteria were analyzed separately. The Gram stain had a sensitivity of $36.1 \%$ (30 of 83) for Gram-positive bacteria and $53.3 \%$ (eight of 15) for Gram-negative bacteria. The specificity was 99.9\% (1636 of 1637) for Gram-positive bacteria and 100\% (1637 of 1637) for Gram-negative bacteria.

Among the 22 patients with ED-BM, there were 13 $(59.1 \%)$ with one or more positive Gram stain findings during the period of ED-BM. Of the eight patients with colonization of the drainage catheter, five $(62.5 \%)$ had one or 


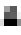
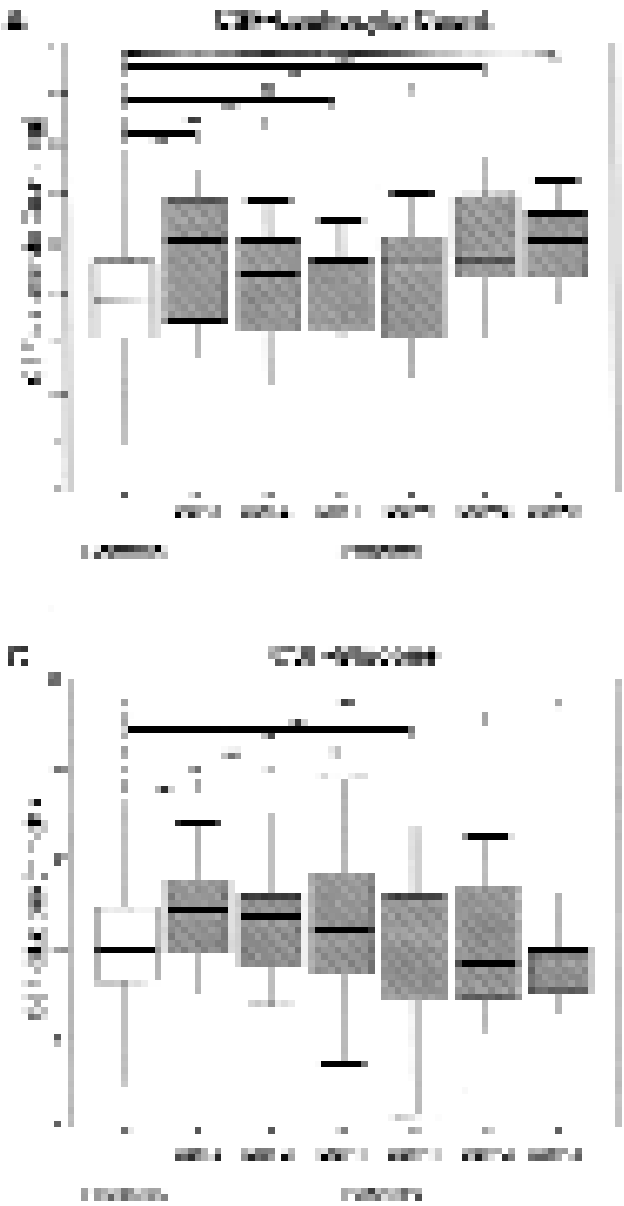

-

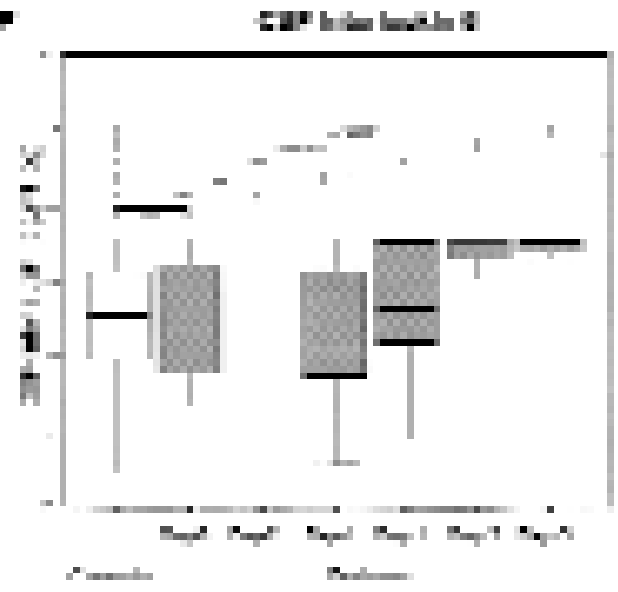

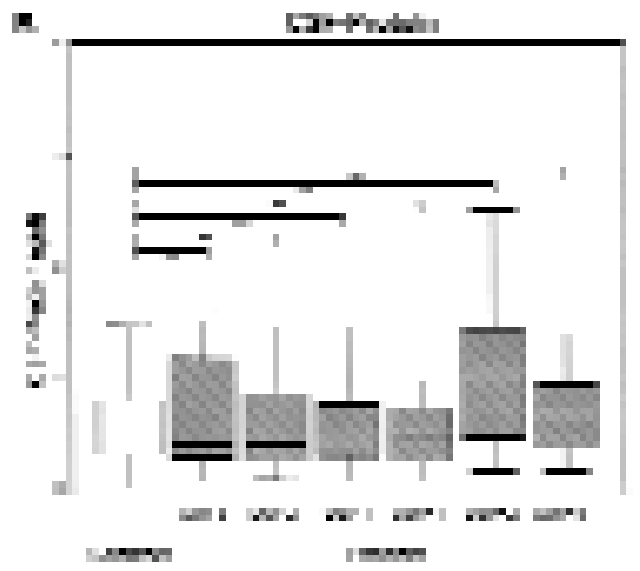

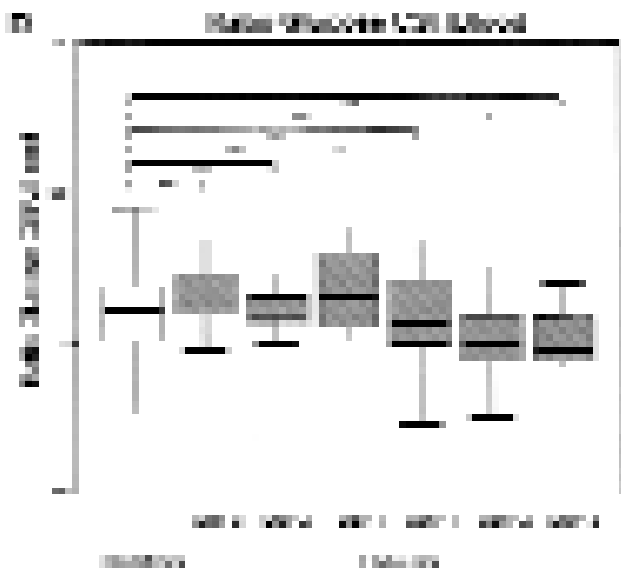

FIG. 2. Graphs showing median measurements of CSF leukocyte count (A), CSF protein concentration (B), CSF glucose concentration (C), CSF/blood glucose ratio (D), and CSF IL-6 concentration $(\mathrm{E})$ in patients with external drains with ED-BM for the 3 days before and 3 days after infection and in control patients without ED-BM. more positive Gram stains. The sensitivity of the Gram stain on the 1st day of infection, that is, the 1st day that the CSF culture was positive, was low: a positive Gram stain was found for only four (18.2\%) of the 22 patients. Three (75\%) of these patients were infected with Gram-positive bacteria, and one (25\%) was infected with Gram-negative bacteria. On the 2nd day of infection, a positive Gram stain was found for nine $(60 \%)$ of 15 patients with a positive CSF culture, and on the 3rd day for six $(60 \%)$ of 10 patients with a positive CSF culture.

\section{Discussion}

We analyzed the predictive and diagnostic value of routine CSF analysis in a cohort of 230 consecutive patients treated with external drainage in whom daily CSF samples were obtained. The results showed that leukocyte count and glucose and protein concentrations in the CSF of external drainage-treated patients with ED-BM were essentially comparable to those in patients with external drains without ED-BM. In both groups, the results of the CSF analyses 

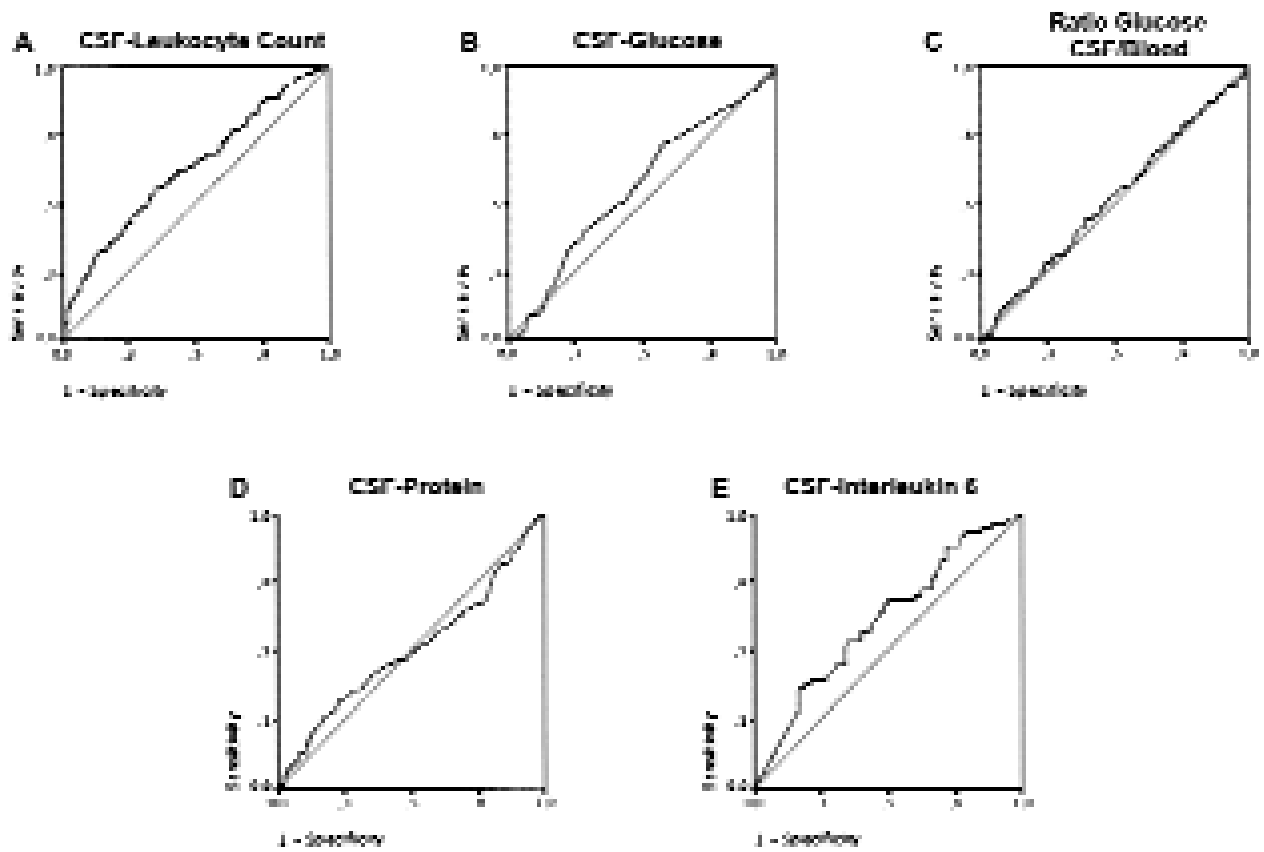

FIG. 3. Graphs showing ROC curves for CSF leukocyte count (A), CSF glucose concentration (B), CSF/blood glucose ratio (C), CSF protein concentration (D), and CSF IL-6 concentration (E) on the 1st day of infection in 22 patients with ED-BM. The ROC curves show all possible combinations of sensitivity and specificity that can be achieved for the parameter.

were heterogeneous during the period of external drainage. When the results obtained in patients with ED-BM during the first 3 days of active infection were compared with the results of the control group without ED-BM, no statistically significant differences were found (Fig. 2). The same was true when the CSF results obtained in patients with ED-BM for the 3 days preceding an active infection were compared with those of the control group.

It is evident that in patients who had recently undergone neurosurgery, the content of CSF is abnormal. Chemical irritation resulting from the presence of blood or blood degradation products in the CSF leads to chemical or aseptic meningitis, with disturbance of the glucose and protein content of the CSF and an increased CSF white blood cell count. ${ }^{3,619}$ As the blood is resorbed from the CSF and the aseptic meningitis subsides, the chemical disturbance normalizes. It is therefore to be expected that the CSF parameters will change, that is, improve, during the period of external drainage in patients in whom bacterial meningitis does not develop. For this reason, we controlled the analysis of the CSF parameters for duration of external drainage by using a matched case-control study. Using univariate regression analysis and ROC curves, we were unable to recognize a significant predictive or diagnostic value for any of the commonly used CSF parameters. More important, we also failed to predict or recognize an infection when results for the same patient were analyzed longitudinally. Thus, detection of even a clear change in CSF parameters in an individual patient did not have a significant predictive value. Using the results for the 200 patients with external drains but who did not have ED-BM as a reference, we found that only a small proportion of the patients in whom ED-BM developed had abnormal values for more than one of the commonly analyzed CSF parameters shortly before or during the course of the ED-BM infection. Therefore, combining the results for different CSF parameters did not increase the predictive or diagnostic value of the CSF analysis.

Although analysis of CSF is often performed routinely in neurosurgery patients, its predictive value for ED-BM has been scarcely studied. Pfisterer and colleagues ${ }^{18}$ performed a transversal analysis of CSF results for 130 patients with external drainage from whom daily CSF samples were obtained. The leukocyte counts were heterogeneous in both the patient group and the control group. Although the authors reported a significant correlation between CSF leukocyte count and CSF infection, there was no clear difference in leukocyte counts between the patients with ED-BM and those without ED-BM. Unfortunately, glucose and protein levels were not analyzed in their report. Pfausler and colleagues ${ }^{17}$ recently suggested that a "cell index" consisting of the ratio of leukocyte and erythrocyte counts in CSF and peripheral blood might be predictive of ED-BM in patients with external drainage. In this pilot study, seven of 13 patients suffered from ED-BM, and the cell index gradually increased in these patients over the 3 days before a conventional diagnosis of ED-BM was made. The cell counts were not adjusted for duration of external drainage, however, and the definition of ED-BM was based on an increase in CSF cell counts. Furthermore, 54\% of the patients with external drains suffered from ED-BM, a percentage that is three to seven times higher than that reported in the literature. ${ }^{4,8,13,14 \text {, }}$ 20,21,25 It is therefore difficult to apply their results to general practice. We used the cell index parameter, as defined by Pfaulser, et al., to analyze the data from our cohort, but we failed to demonstrate a predictive or diagnostic value for ED-BM (data not shown).

It has been suggested that determination of CSF cytokine levels may be helpful in the diagnosis of bacterial meningi- 
Analysis of CSF in drainage-related bacterial meningitis

TABLE 1

Predictive and diagnostic value of abnormal CSF parameters for ED-BM in a matched case-control study*

\begin{tabular}{|c|c|c|c|c|c|c|c|c|}
\hline \multirow[b]{2}{*}{ Variable } & \multicolumn{8}{|c|}{ No./Total (\%) } \\
\hline & Patients & $\begin{array}{c}\text { Control } \\
\text { Volunteers }\end{array}$ & Patients & $\begin{array}{c}\text { Control } \\
\text { Volunteers }\end{array}$ & Patients & $\begin{array}{c}\text { Control } \\
\text { Volunteers }\end{array}$ & Patients & $\begin{array}{c}\text { Control } \\
\text { Volunteers }\end{array}$ \\
\hline $\begin{array}{l}\text { no. of ab- } \\
\text { normal pa- } \\
\text { rameters }\end{array}$ & 1 & 1 & 2 & 2 & 3 & 3 & 4 & 4 \\
\hline \multicolumn{9}{|l|}{ Day $\dagger$} \\
\hline-3 & 4/16 (25) & 2/12 (17) & $0 / 16(0)$ & $1 / 12(8)$ & $0 / 16(0)$ & $0 / 12(0)$ & $0 / 16(0)$ & $0 / 12(0)$ \\
\hline-2 & $2 / 14$ (14) & $3 / 13(23)$ & $0 / 14(0)$ & $0 / 13(0)$ & $0 / 14(0)$ & $0 / 13(0)$ & $0 / 14(0)$ & $0 / 13(0)$ \\
\hline-1 & $1 / 13(8)$ & $4 / 15(27)$ & $1 / 13(8)$ & $2 / 15$ (13) & $0 / 13(0)$ & $1 / 15(7)$ & $0 / 13(0)$ & $0 / 15(0)$ \\
\hline+1 & 4/20 (20) & 4/13 (31) & $2 / 20(10)$ & $1 / 13(8)$ & $1 / 20(5)$ & 0/13 (0) & $0 / 20(0)$ & $0 / 13(0)$ \\
\hline+2 & $6 / 16(38)$ & $2 / 13(15)$ & $5 / 16(31)$ & $0 / 13(0)$ & $1 / 16(6)$ & $0 / 13(0)$ & $0 / 16(0)$ & $0 / 13(0)$ \\
\hline+3 & 4/11 (36) & 2/15 (13) & $1 / 11(9)$ & $1 / 15$ (7) & $0 / 11(0)$ & $0 / 15(0)$ & $0 / 11(0)$ & $0 / 15(0)$ \\
\hline
\end{tabular}

* Abnormal values were above the 95th percentile for both CSF leukocyte count and protein concentration and below the 5th percentile for CSF glucose concentration and CSF/blood glucose ratio in 994 CSF samples from 200 patients with external drainage but without ED-BM. There were no statistically significant differences between patients and control volunteers ( $\mathrm{p}>0.05$, McNemar test).

$\dagger$ Indicates days before $(-)$ or after $(+)$ infection.

tis. ${ }^{10,12}$ Lopez-Cortes and colleagues ${ }^{11}$ found that CSF levels of tumor necrosis factor- $\alpha$, IL-1 $\beta$, IL-6, and IL- 8 were significantly higher in neurosurgical patients with bacterial meningitis than in patients with asymptomatic aseptic meningitis. These inflammatory cytokines are synthesized intrathecally by activated leukocytes ${ }^{7,24}$ and were found to be more specific predictors of bacterial meningitis, compared with cell counts, in the study by Lopez-Cortes and colleagues. We analyzed the predictive and diagnostic value of IL-6 in neurosurgical patients with external drains. Our results of a group of $166 \mathrm{CSF}$ samples showed that IL-6 levels in CSF were closely correlated with cell counts. We found no predictive value of IL-6 in CSF for ED-BM. We did find that in the CSF of patients with external drains and active bacterial meningitis, IL-6 levels were significantly higher than in the CSF of patients with external drains and no ED-BM, but we could not establish the diagnostic value in a matched case-control analysis.

We also evaluated the diagnostic value of the daily Gram stain in our cohort. We found that a large proportion of the patients with ED-BM did not have positive Gram stain findings during the period of positive CSF cultures. Positive Gram stain results were found for only $60 \%$ of the patients with ED-BM on 1 or more days during active infection. In an evaluation of the use of the Gram stain as a screening tool, we found that the Gram stain was positive for only $20 \%$ of the patients with ED-BM on the 1st day a positive CSF culture was obtained. The Gram stain proved to be very specific $(\sim 100 \%)$ and to have a high positive predictive value (97\%) in this cohort. These findings suggest that the Gram stain can be used as a diagnostic tool in patients with external drains in whom ED-BM is suspected.

The clinical features of ED-BM may be difficult to distinguish from symptoms that frequently occur in patients in the intensive care unit. Patients with intracranial hemorrhage or serious head injury may show signs of aseptic meningitis, such as headache, nausea, and neck stiffness. ${ }^{2,13}$ In patients in the intensive care unit who receive ventilation and sedation, the evaluation of clinical signs and the detection of a new focal neurological deficit or changes in mental status can be very difficult. For these reasons, guidelines often recommend that in cases of possible ED-BM, CSF analysis should be performed, and in cases of a clear deterioration of the CSF chemistry and CSF leukocyte count, ED$\mathrm{BM}$ is highly suspected.,13

\section{Conclusions}

The results of this study showed that the commonly analyzed CSF parameters have no predictive value for ED-BM, even when several parameters are considered in combination. Thus, the routine use of chemical analysis of CSF samples to screen patients with external drains for ED-BM has no additional value. Also, the analysis of an isolated CSF sample in a patient in whom ED-BM is highly suspected has no additional value, due to the absence of clear diagnostic cutoff levels. The diagnosis of ED-BM can therefore be based only on the results of microbiological cultures. ${ }^{2,13}$ In our hospital, daily CSF samples are obtained in patients with external drains to evaluate microbial growth in the CSF. One might argue that to reduce unnecessary manipulation of drains and the accompanying risk of infection, less frequent sampling, that is, every other day, might be sufficient. The risk of infection related to external drainage is very low during the 1st days of drainage and increases with every day the drain is in place. ${ }^{13,20}$ Therefore, we suggest a sample regimen of two times a week during the 1st week of drainage and daily sampling during the rest of the drainage period.

\section{Acknowledgments}

We thank Marja Kersbergen-van Oostrom and Margo van SchieTroost for assistance in performing the IL-6 measurements.

\section{References}

1. Acikbas SC, Akyuz M, Kazan S, Tuncer R: Complications of closed continuous lumbar drainage of cerebrospinal fluid. Acta Neurochir (Wien) 144:475-480, 2002

2. Anonymous: The management of neurosurgical patients with postoperative bacterial or aseptic meningitis or external ventricular drain-associated ventriculitis. Infection in Neurosurgery Working Party of the British Society for Antimicrobial Chemotherapy. Br J Neurosurg 14:7-12, 2000 
3. Blomstedt GC: Post-operative aseptic meningitis. Acta Neurochir (Wien) 89:112-116, 1987

4. Bogdahn U, Lau W, Hassel W, Gunreben G, Mertens HG, Brawanski A: Continuous-pressure controlled, external ventricular drainage for treatment of acute hydrocephalus - evaluation of risk factors. Neurosurgery 31:898-904, 1992

5. Coplin WM, Avellino AM, Kim DK, Winn HR, Grady MS: Bacterial meningitis associated with lumbar drains: a retrospective cohort study. J Neurol Neurosurg Psychiatry 67:468-473, 1999

6. Forgacs P, Geyer CA, Freidberg SR: Characterization of chemical meningitis after neurological surgery. Clin Infect Dis 32: 179-185, 2001

7. Frei K, Malipiero UV, Leist TP, Zinkernagel RM, Schwab ME, Fontana A: On the cellular source and function of interleukin 6 produced in the central nervous system in viral diseases. Eur $\mathbf{J}$ Immunol 19:689-694, 1989

8. Holloway KL, Barnes T, Choi S, Bullock R, Marshall LF, Eisenberg HM, et al: Ventriculostomy infections: the effect of monitoring duration and catheter exchange in 584 patients. J Neurosurg 85:419-424, 1996

9. Kitchel SH, Eismont FJ, Green BA: Closed subarachnoid drainage for management of cerebrospinal fluid leakage after an operation on the spine. J Bone Joint Surg (Am) 71:984-987, 1989

10. Lopez-Cortes LF, Cruz-Ruiz M, Gomez-Mateos J, JimenezHernandez D, Palomino J, Jimenez E: Measurement of levels of tumor necrosis factor-alpha and interleukin-1 beta in the CSF of patients with meningitis of different etiologies: utility in the differential diagnosis. Clin Infect Dis 16:534-539, 1993

11. Lopez-Cortes LF, Marquez-Arbizu R, Jimenez-Jimenez LM, Jimenez-Mejias E, Caballero-Granado FJ, Rey-Romero C, et al: Cerebrospinal fluid tumor necrosis factor-alpha, interleukin-1beta, interleukin-6, and interleukin-8 as diagnostic markers of cerebrospinal fluid infection in neurosurgical patients. Crit Care Med 28:215-219, 2000

12. Lorino G, Angeletti S, Gherardi G, De Cesaris M, Gherardi L, Battistoni F, et al: Diagnostic value of cytokine assays in cerebrospinal fluid in culture-negative, polymerase chain reaction-positive bacterial meningitis. Eur J Clin Microbiol Infect Dis 19: 388-392, 2000

13. Lozier AP, Sciacca RR, Romagnoli MF, Connolly ES Jr: Ventriculostomy-related infections: a critical review of the literature. Neurosurgery 51:170-182, 2002

14. Lyke KE, Obasanjo OO, Williams MA, O'Brien M, Chotani $\mathrm{R}$, Perl TM: Ventriculitis complicating use of intraventricular catheters in adult neurosurgical patients. Clin Infect Dis 33: 2028-2033, 2001

15. Mayhall CG, Archer NH, Lamb VA, Spadora AC, Baggett JW,
Ward JD, et al: Ventriculostomy-related infections. A prospective epidemiologic study. N Engl J Med 310:553-559, 1984

16. Murray PR, Baron EJ, Pfaller MA, Tenover FC, Yolken RH: Manual of Clinical Microbiology, ed 7. Washington DC: AJM Press, 1999

17. Pfausler B, Beer R, Engelhardt K, Kemmler G, Mohsenipour I, Schmutzhard E: Cell index - a new parameter for the early diagnosis of ventriculostomy (external ventricular drainage)-related ventriculitis in patients with intraventricular hemorrhage? Acta Neurochir (Wien) 146:477-481, 2004

18. Pfisterer W, Muhlbauer M, Czech T, Reinprecht A: Early diagnosis of external ventricular drainage infection: results of a prospective study. J Neurol Neurosurg Psychiatry 74:929-932, 2003

19. Ross D, Rosegay H, Pons V: Differentiation of aseptic and bacterial meningitis in postoperative neurosurgical patients. J Neurosurg 69:669-674, 1988

20. Schade RP, Schinkel J, Visser LG, Van Dijk JMC, Voormolen JHC, Kuijper EJ: Bacterial meningitis caused by the use of ventricular or lumbar cerebrospinal fluid catheters. J Neurosurg 102: 229-234, 2005

21. Schultz M, Moore K, Foote AW: Bacterial ventriculitis and duration of ventriculostomy catheter insertion. J Neurosci Nurs 25: 158-164, 1993

22. Shapiro SA, Scully T: Closed continuous drainage of cerebrospinal fluid via a lumbar subarachnoid catheter for treatment or prevention of cranial/spinal cerebrospinal fluid fistula. Neurosurgery 30:241-245, 1992

23. Sundbarg G, Nordstrom CH, Soderstrom S: Complications due to prolonged ventricular fluid pressure recording. Br J Neurosurg 2:485-495, 1988

24. Van Meir E, Ceska M, Effenberger F, Walz A, Grouzmann E, Desbaillets I, et al: Interleukin-8 is produced in neoplastic and infectious diseases of the human central nervous system. Cancer Res 52:4297-4305, 1992

25. Winfield JA, Rosenthal P, Kanter RK, Casella G: Duration of intracranial pressure monitoring does not predict daily risk of infectious complications. Neurosurgery 33:424-431, 1993

Manuscript received January 5, 2005.

Accepted in final form August 22, 2005.

This study was funded by a grant from the efficacy research program at Leiden University Medical Center.

Address reprint requests to: Rogier P. Schade, M.D., Ph.D., Department of Medical Microbiology (440 MMB), Radboud University Nijmegen Medical Center, Box 9101, 6500 HB Nijmegen, The Netherlands.email: r.schade@mmb.umcn.nl. 